\title{
Un experimento para ilustrar el primer principio de la termodinámica en bachillerato: creación de una nube de alcohol dentro de una botella
}

\author{
Antonio Tomás-Serrano \\ Departamento de Física. Universidad de Murcia.España.atserrano@gmail.com
}

[Recibido: 30 diciembre 2020. Revisado: 29 marzo 2021. Aceptado: 29 marzo 2021]

Resumen: En este artículo se propone la utilización de un sencillo experimento (la creación de una nube de alcohol) como recurso didáctico para el estudio de la primera ley de la termodinámica en bachillerato, aunque también puede utilizarse en otros contextos y en diferentes niveles educativos. Teniendo en cuenta el alumnado al que se dirige esta propuesta, se realiza un análisis, esencialmente, cualitativo de los procesos físicos que intervienen en este experimento (evaporación-condensación de un líquido y compresión-expansión de un gas). Además, se comenta el posible uso de otros líquidos (especialmente, el agua) y se plantean varios experimentos complementarios que ayudan a comprender por qué se forma la nube.

Palabras clave: Primer principio de la termodinámica; Proceso adiabático; Presión de vapor; Nube de alcohol.

An experiment to illustrate the first principle of thermodynamics in high school: creating a cloud of alcohol inside a bottle Abstract: This article proposes to conduct a simple experiment as a didactic resource for the study of the first
law of thermodynamics in high school, although it can also be used in other contexts and at different educational
levels. Taking into account the students to whom this proposal is intended, an essentially qualitative analysis of
the physical processes involved in this experiment (evaporation-condensation of a liquid and compression-
expansion of a gas) is carried out. In addition, the possible use of other liquids (especially, water) to carry out the
experiment is discussed and several complementary experiments are proposed that help to understand why the
cloud is formed.

Keywords: First law of thermodynamics; Adiabatic process; Vapour pressure; Alcohol cloud.

Para citar este artículo: Tomás-Serrano A. (2021) Un experimento para ilustrar el primer principio de la termodinámica en bachillerato: creación de una nube de alcohol dentro de una botella. Revista Eureka sobre Enseñanza y Divulgación de las Ciencias 18(3), 3401. doi: 10.25267/Rev_Eureka_ensen_divulg_cienc.2021.v18.i3.3401

\section{Introducción}

Los conceptos de calor, trabajo y energía se estudian en diferentes niveles de la enseñanza secundaria, pero no se profundiza en la relación entre ellos hasta el bachillerato, cuando se aborda el primer principio de la termodinámica.

En la bibliografía podemos encontrar numerosas referencias acerca de las dificultades que encuentran los estudiantes para comprender el significado del primer principio, ya sea por la complejidad de los conceptos que relaciona (Feynman, Leighton y Sands 1971, Holton 1984, Rozier y Viennot 1990, Pintó 1991), o por la forma en que se suele presentar sus contenidos en los libros de texto (Furió, Solbes y Furió 2007, Álvarez y Manzano 2018). Y es que, a pesar de su aparente simplicidad - una nueva formulación de la conservación de la energía-, la aplicación del primer principio de la termodinámica «permite alcanzar importantes conclusiones cuantitativas que no se derivan de forma inmediata de su enunciado» (Barrow 1985). De hecho, el establecimiento de esta ley costó mucho porque la gran mayoría de los hechos experimentales conocidos hasta bien entrado el siglo XIX se podían explicar con la teoría del calórico (Kane y Sternheim 1996). 
Por otra parte, como es difícil encontrar en los manuales de prácticas experimentos que faciliten la comprensión de estos conceptos y sus relaciones, el estudio de la termodinámica suele quedar reducido al plano puramente teórico.

Por todo ello, algunos autores (Cortel 2004, Güemez, Fiolhais y Fiolhais 2007, Kamcharean,

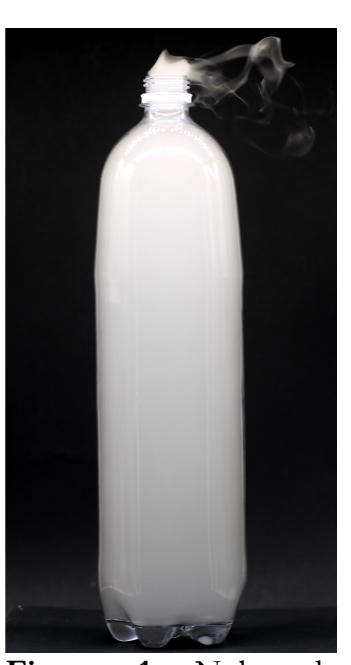

Figura 1. Nube de alcohol dentro de una botella. Khanchong y Wattanakasiwich 2016) proponen ilustrar la aplicación de los conceptos básicos de termodinámica mediante experimentos sencillos, tales como el pistón de ignición o la creación de una nube de agua dentro de una botella. La propuesta de trabajo que se presenta a continuación está directamente relacionada con este último, aunque se sustituye el agua por el etanol para conseguir resultados más llamativos, un aspecto a tener en cuenta cuando el experimento va dirigido a alumnos de niveles no universitarios (figura 1).

Dada su sencillez y vistosidad, este experimento se presenta normalmente como una demostración dirigida al público en general e, incluso, a niños (Spangler 2017); no obstante, su análisis en profundidad revela matices que resultan muy adecuados para reflexionar, con nuestros alumnos, sobre el concepto de trabajo de expansióncompresión, las diferencias entre procesos reversibles e irreversibles, las características de los procesos adiabáticos e isotérmicos, los procesos de evaporación y condensación, etc.

\section{Formación de una nube de etanol}

Para llevar a cabo este experimento se necesitarán los materiales mostrados en la figura 2: una pequeña cantidad de etanol (puede utilizarse alcohol de uso sanitario o cosmético), un pulverizador (opcional), una botella de refresco de politereftalato de etileno (en adelante, PET) transparente, con una capacidad comprendida entre 1 L y 2 L (las botellas de agua no son adecuadas porque están diseñadas para soportar presiones inferiores a las de las botellas de refresco), un tapón de corcho que ajuste bien en la boca de la botella, una aguja para inflar balones y una bomba para bicicleta, preferiblemente con manómetro. La aguja debe atravesar el tapón (figura 2), de tal modo que ajuste lo mejor posible para minimizar fugas de aire cuando aumente la presión dentro de la botella.

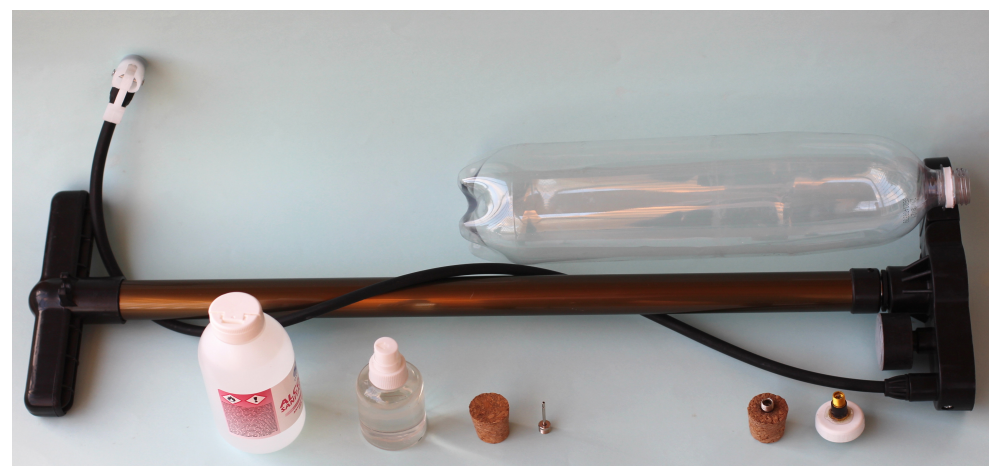

Figura 2. Materiales necesarios para realizar el experimento: bomba de bicicleta con manómetro, alcohol de $96^{\circ}$, pulverizador, botella de gaseosa fabricada con PET, tapón de corcho y aguja de inflar balones. En la parte inferior derecha se muestran dos posibles acoplamientos para inyectar aire a la botella: una aguja de inflar balones que atraviesa un tapón de corcho y una válvula de neumático insertada en el tapón de la botella y fijada con adhesivo.

El experimento consiste en introducir unos $20 \mathrm{~cm}^{3}$ de etanol en la botella (la cantidad no es crítica), ajustar el tapón (con la aguja acoplada) y agitar la botella durante unos segundos para que se evapore la máxima cantidad posible de alcohol. También se puede conseguir este 
resultado utilizando un pequeño atomizador para introducir $1 \mathrm{~cm}^{3}$ de alcohol pulverizado. Seguidamente, se conecta el cabezal de la bomba a la aguja y se retiene con una mano, mientras se bombea aire hasta que la presión indicada por el manómetro sea de unos 2 bar $\left(2 \times 10^{5} \mathrm{~Pa}\right.$ sobre la presión atmosférica; este tipo de manómetros suele estar graduado en bares), es decir, una presión absoluta de 3 bar. Esta operación dura menos de un minuto y requiere de 15 a 20 carreras del émbolo de una bomba de $70 \mathrm{~cm}$ de longitud, como la que aparece en la figura 2.

Alcanzada la presión deseada, se sujeta el cuerpo de la botella con la mano que accionaba la bomba y, con la otra, se libera el tapón para que se expandan rápidamente el aire y el vapor de etanol (generalmente, el tapón saltará bruscamente hacia arriba en cuanto se deje de sujetar). A consecuencia de esta expansión, se enfriará la botella y se formará una nube de etanol, como la mostrada en la figura 1, que se irá desvaneciendo a medida que vuelva a aumentar la temperatura en el interior del recipiente. Por este motivo, si antes de que desaparezca la nube se vuelve a colocar el tapón y se bombea aire al interior, la nube se desvanecerá rápidamente. Este ciclo de compresión-descompresión, con la consiguiente desaparición-formación de la nube, puede repetirse indefinidamente mientras quede alcohol en fase líquida dentro de la botella.

\section{¿Por qué se forma la nube?}

A una temperatura dada, la rapidez de evaporación depende del área de la superficie del líquido en contacto con el aire contenido en la botella. Por esta razón, es aconsejable introducir el alcohol pulverizado, o agitar la botella, para aumentar la superficie a través de la cual ocurre la evaporación. Como este proceso es endotérmico, la temperatura del interior de la botella disminuye inicialmente, pero al ser muy pequeña la cantidad de etanol que se evapora, se puede ignorar este descenso en una primera aproximación. Transcurridos unos minutos, se alcanzará una situación de equilibrio dinámico en el que la temperatura y la presión de vapor del etanol permanecerán constantes y el aire dentro de la botella estará saturado en etanol.

Si una vez alcanzado el equilibrio a temperatura ambiente, se provoca un súbito enfriamiento, la presión de vapor de equilibrio a esta nueva temperatura será inferior a la del equilibrio inicial. La consecuencia es que habrá un exceso de etanol, en fase vapor, que condensará bruscamente y formará una nube de diminutas gotas que dispersan todas las frecuencias de la luz (blanca) incidente (Fishbane et al. 1993). Obviamente, si esta caída de temperatura ocurre antes de que se haya alcanzado la saturación, habrá menor cantidad de vapor de etanol que pueda condensar y la nube formada tendrá menor densidad.

La bajada de temperatura se puede llevar a cabo introduciendo la botella en un congelador, pero se consiguen mejores resultados mediante una rápida descompresión del sistema; esta es la razón por la que hay que bombear aire previamente al interior de la botella. Debido a la introducción de este aire comprimido, la temperatura aumenta entre $5^{\circ} \mathrm{C}$ y $8{ }^{\circ} \mathrm{C}$, en función de la presión máxima alcanzada, de la rapidez con que se bombea y del tamaño de la botella.

Veamos qué ocurre cuando la mezcla de aire y de vapor de etanol (sistema en estudio) es sometida a una descompresión y realiza un trabajo $W$ sobre la atmósfera (entorno del sistema).

Si la expansión es muy rápida, de modo que prácticamente no hay intercambio de calor entre el sistema y su entorno $(Q=0)$, se puede considerar que el proceso es adiabático. Si se adopta el convenio de considerar positivos al calor cedido (por el entorno) al sistema y al trabajo realizado por el sistema (sobre su entorno), la variación de energía interna $U$ del sistema vendrá dada por 


$$
\Delta U=Q-W,
$$

y, como en este caso $Q=0$, quedará

$$
\Delta U=-W,
$$

es decir, el sistema realiza trabajo sobre la atmósfera a expensas de su energía interna. Esta disminución de energía interna se traducirá en una disminución de la temperatura de los gases que se expanden; si la caída de temperatura que ocurre es suficientemente grande, condensará bruscamente la mayor parte del etanol evaporado y formará una nube. No obstante, como el sistema no está aislado del entorno, su temperatura ascenderá rápidamente y la nube se disipará al evaporarse nuevamente el etanol condensado.

\section{Temperatura mínima tras la descompresión}

A pesar de que la turbulencia, generada en el cuello de la botella durante la descompresión, absorbe una cantidad de energía que es muy difícil de cuantificar, se puede establecer una cota inferior para la temperatura que alcanza este sistema suponiendo que los $n$ moles de gas (aire y vapor de etanol) que contiene la botella, tras la operación de bombeo, se comportan idealmente y sufren una expansión adiabática irreversible (Güemez et al. 2007) desde su estado inicial, en el que $p_{1}=3,0 \times 10^{5} \mathrm{~Pa}, V_{1}=1,5 \times 10^{-3} \mathrm{~m}^{3}, T_{1}=300 \mathrm{~K}$, hasta un estado final en el que ocupan un volumen $V_{2}$ a una temperatura $T_{2}$ y presión atmosférica $p_{2}=1,0 \times 10^{5} \mathrm{~Pa}$. Para simplificar los cálculos, y dado que el número de moles de vapor de etanol $n$ es mucho menor que el de aire $n_{\text {aire }}$, consideraremos que el número total de moles $n$ es igual a los del aire $(n=$ $\left.n_{\text {aire }}+n_{\mathrm{e}} \approx n_{\text {aire }}\right)$.

La variación de energía interna de los $n$ moles de aire viene dada por

donde $n$ vale

$$
\Delta U=n C_{v}\left(T_{2}-T_{1}\right) \text {, }
$$

$$
n=\frac{p_{1} V_{1}}{R T_{1}}=\frac{3,0 \times 10^{5} \times 1,5 \times 10^{-3}}{8,314 \times 300}=0,18 \mathrm{~mol},
$$

siendo $R$ la constante universal de los gases $\left(8,314 \mathrm{~J} \cdot \mathrm{mol}^{-1} \cdot \mathrm{K}^{-1}\right)$ y $C_{v}$ la capacidad calorífica del aire a volumen constante $(5 \mathrm{R} / 2)$.

Por otra parte, el trabajo realizado por este aire al expandirse, contra la presión constante de la atmósfera, viene dado por

$$
W=p_{2}\left(V_{2}-V_{1}\right),
$$

donde

$$
V_{2}=\frac{n R T_{2}}{p_{2}}
$$

Sustituyendo las ecuaciones (3), (5) y (6) en la ecuación (2), se encuentra para la temperatura final $T_{2}$

$$
T_{2}=\frac{p_{2} V_{1}+n C_{v} T_{1}}{n\left(C_{v}+R\right)} \approx 243 \mathrm{~K}\left(=-30^{\circ} \mathrm{C}\right),
$$

es decir, la temperatura del sistema podría descender hasta $-30{ }^{\circ} \mathrm{C}\left(\Delta T \approx-57^{\circ} \mathrm{C}\right)$.

\section{Estimación de la cantidad máxima de etanol que puede condensar}


Se puede calcular aproximadamente la masa de etanol que condensa para formar la nube si se admite que su vapor se comporta idealmente en las condiciones de presión y temperatura en las que se realiza este experimento y, por otra parte, se alcanza rápidamente el equilibrio de saturación a la temperatura de trabajo. Bajo estas suposiciones, el número de moles de etanol evaporado $n$ errá igual a

$$
n_{\mathrm{e}}=\frac{p_{\mathrm{e}} V_{1}}{R T_{1}},
$$

donde $p_{\mathrm{e}}$ es la presión de vapor del etanol en el equilibrio, a la temperatura de trabajo $T_{1}(300$ $\mathrm{K})$ y $V_{1}$ es el volumen que ocupa dicho vapor $(1,5 \mathrm{~L})$. La masa de etanol evaporado $m_{\mathrm{e}}$ será el producto de su masa molar $M\left(46 \mathrm{~g} \cdot \mathrm{mol}^{-1}\right)$ por el número de moles de etanol evaporado

$$
m_{\mathrm{e}}=M \cdot n_{\mathrm{e}} .
$$

Para hacer una primera estimación de esta masa de etanol se puede utilizar una tabla de presión de vapor (como la Tabla 1) o la correspondiente gráfica de $p_{\mathrm{e}}$ frente a $T$ (figura 3).

Tabla 1. Presión de vapor del etanol a diferentes temperaturas; datos tomados de DDB (2020).

\begin{tabular}{|l|c|c|c|c|c|c|c|c|c|c|c|c|c|c|c|c|}
\hline$T(\mathrm{~K})$ & 238 & 243 & 248 & 253 & 258 & 263 & 268 & 273 & 278 & 283 & 288 & 293 & 298 & 303 & 308 & 313 \\
\hline$T\left({ }^{\circ} \mathrm{C}\right)$ & -35 & -30 & -25 & -20 & -15 & -10 & -5 & 0 & 5 & 10 & 15 & 20 & 25 & 30 & 35 & 40 \\
\hline$P_{\mathrm{c}}(\mathrm{kPa})$ & 0,08 & 0,13 & 0,21 & 0,33 & 0,50 & 0,74 & 1,09 & 1,59 & 2,22 & 3,14 & 4,28 & 5,83 & 7,87 & 10,5 & 13,4 & 17,9 \\
\hline
\end{tabular}

En la gráfica de la figura 3 se observa que si se establece el equilibrio de saturación del vapor a $300 \mathrm{~K}$ (punto $1, p_{\mathrm{e}, 300}$ ), la presión de vapor del etanol será, aproximadamente, $9 \times 10^{3} \mathrm{~Pa} \mathrm{y}$, según las ecuaciones (8) y (9), la masa de etanol evaporado a dicha temperatura $m_{\mathrm{e}, 300}$ valdrá

$$
m_{\mathrm{e}, 300} \simeq \frac{46 \times 9 \times 10^{3} \times 1,5 \times 10^{-3}}{8,314 \times 300}=0,25 \mathrm{~g} .
$$

Procediendo del mismo modo, se encuentra que la presión de vapor del etanol a la temperatura mínima que se podría alcanzar tras la descompresión, $243 \mathrm{~K}$ (punto 2 de la gráfica $\left.3, p_{\mathrm{e}, 243}\right)$, y la masa correspondiente de vapor de etanol en equilibrio a dicha temperatura $m_{\mathrm{e}, 243}$ son, respectivamente, $130 \mathrm{~Pa}$ y $4,4 \times 10^{-3} \mathrm{~g}$.

Dado que la masa de etanol que puede permanecer en fase vapor a $243 \mathrm{~K}$ es bastante menor que la masa de vapor en el equilibrio a $300 \mathrm{~K}$, condensará la práctica totalidad del etanol evaporado (aproximadamente, el $98 \%$ ).

Se puede obtener un valor más exacto de la presión de vapor mediante interpolación lineal en la Tabla 1 o, como se muestra en la siguiente sección, a partir de la ecuación de ClausiusClapeyron.

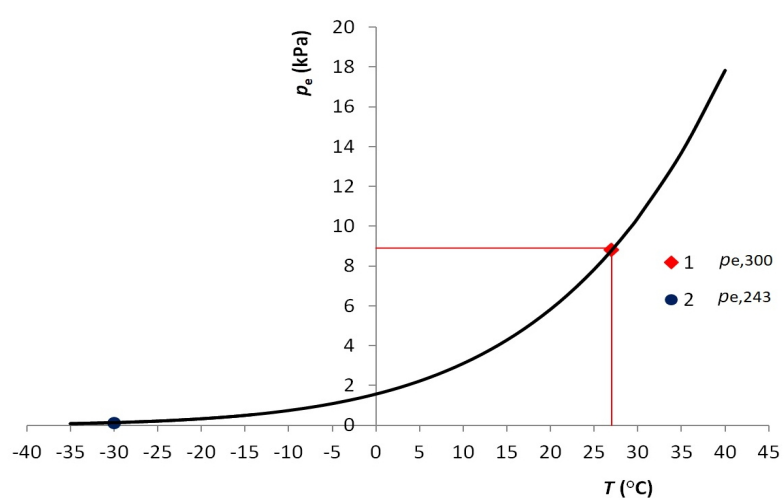

Figura 3. Variación de la presión de vapor del etanol con la temperatura. Los puntos 1 y 2 de la gráfica corresponden a la situación inicial $\left(T_{1}=300 \mathrm{~K}=27^{\circ} \mathrm{C}\right) \mathrm{y}$ final $\left(T_{2}=243 \mathrm{~K}=-30{ }^{\circ} \mathrm{C}\right)$, respectivamente, del experimento descrito. 


\section{Cálculo de la presión de vapor con la ecuación de Clausius-Clapeyron}

La presión de vapor del etanol, a una temperatura $T$, se puede calcular a partir de la ecuación de Clausius-Clapeyron

$$
\ln \left(\frac{p_{e}}{p_{0}}\right)=\frac{\Delta H_{v}}{R}\left(\frac{1}{T_{0}}-\frac{1}{T}\right),
$$

donde $p_{0}$ es la presión de vapor a una temperatura $T_{0} p_{0}$ y $T_{0}$ son valores de referencia conocidos) e $\Delta H_{v}$ es la entalpía de vaporización del etanol, supuesta constante $\left(43,2 \mathrm{~kJ} \cdot \mathrm{mol}^{-1}\right)$, aunque, en realidad, $\Delta H_{\nu}$ disminuye ligeramente al aumentar la temperatura. En los cálculos que siguen se tomarán como referencias $T_{0}=273 \mathrm{~K} \mathrm{y} p_{0}=p_{273 \mathrm{~K}}=1593 \mathrm{~Pa}$ (DDB 2020), ya que con ellas la ecuación de Clausius-Clapeyron da unos valores para la presión de vapor del etanol que concuerdan razonablemente bien con los que se determinan experimentalmente en el rango de temperaturas que nos interesa en este experimento.

De acuerdo con la ecuación (10), a una temperatura de $300 \mathrm{~K}$, la presión de vapor del etanol será

$$
\ln \left(\frac{p_{\mathrm{e}, 300}}{1593}\right)=\frac{43200}{8,314}\left(\frac{1}{273}-\frac{1}{300}\right) ; \quad p_{\mathrm{e}, 300} \approx 8,8 \times 10^{3} \mathrm{~Pa}
$$

y la correspondiente masa de etanol evaporado $m_{\mathrm{e}, 300}$ valdrá $0,24 \mathrm{~g}$.

Los cálculos anteriores justifican la formación de la nube de etanol siempre y cuando se cumplan las premisas en que se basan, esto es, que en el momento de la descompresión (desde $3 \times 10^{5} \mathrm{~Pa}$ hasta $1 \times 10^{5} \mathrm{~Pa}$ ) se haya alcanzado la saturación del vapor de etanol y que la temperatura del sistema disminuya considerablemente (desde $300 \mathrm{~K}$ a $243 \mathrm{~K}$ ). Sin embargo, en el experimento real no se cumplen rigurosamente estas dos condiciones porque, desde que se introduce el etanol en la botella, hasta que se efectúa la descompresión, transcurre normalmente menos de un minuto, un periodo de tiempo insuficiente para que se alcance la saturación. Por otra parte, para que la temperatura llegase a ser de $243 \mathrm{~K}$, la descompresión debería ser muy rápida y no generarse turbulencias en el cuello de la botella.

Analizaremos con más detalle estas cuestiones a continuación.

\section{¿Qué cantidad de etanol se evapora durante el experimento?}

La masa de etanol evaporado, en el momento previo a la descompresión, se puede determinar a partir de la presión que ejerce el vapor del etanol, en ese instante (ecuaciones 8 y 9), en el interior de la botella (figura 4). Para efectuar esta medida se han insertado dos tubos delgados de PVC flexible en la botella, uno para introducir el etanol (con una jeringa) y otro para conectar un manómetro diferencial y medir el exceso de presión sobre la atmosférica. Estos tubos deben tener un diámetro tal que permita conexiones estancas de la botella con la boquilla de la jeringa y con el manómetro. Para evitar fugas del vapor de etanol mientras se realizan las medidas, la unión de los tubos a la botella debe sellarse cuidadosamente (por ejemplo, con silicona). 


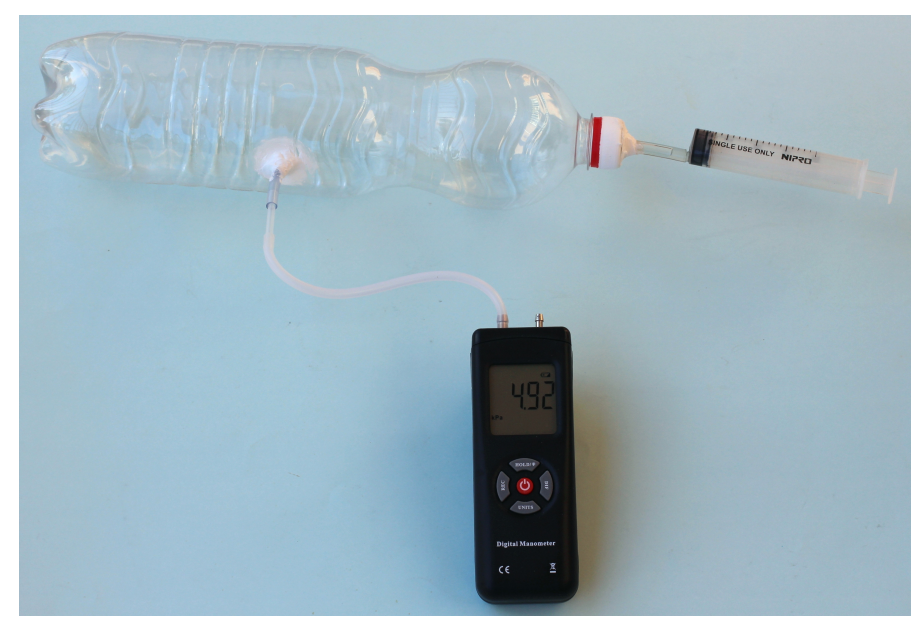

Figura 4. Medida de la presión de vapor del etanol con un manómetro diferencial. El alcohol se introduce con una jeringa a través del tubo insertado en el tapón de la botella.

Los resultados obtenidos en estas medidas, a una temperatura próxima a $300 \mathrm{~K}$ y con unos 20 $\mathrm{cm}^{3}$ de etanol en una botella de 1,5 L, muestran que, si se agita suavemente la botella, a los 30 segundos de la introducción del alcohol, la presión de vapor alcanza, aproximadamente, el 75 $\%$ del valor de la presión en el equilibrio a la temperatura de trabajo, mientras que a los 60 segundos alcanza el $80 \%$ de dicho valor.

Así pues, aunque no se llegue a alcanzar la saturación, la cantidad de vapor que puede condensar tras la descompresión $(0,75 \times 0,24=0,18 \mathrm{~g}$, a $300 \mathrm{~K})$ es suficiente para garantizar la formación de una densa nube de pequeñísimas gotas de etanol.

\section{Medida de la temperatura tras la descompresión}

La formación de la nube de etanol es una prueba evidente de que la temperatura desciende notablemente en el interior de la botella debido a la descompresión que ocurre (Hecht 1998). Sin embargo, cuando se intenta medir esta variación de temperatura con un termómetro, que utiliza como sensor un termopar convencional (figura 5), se detecta una caída relativamente pequeña $\left(\Delta T \approx-10^{\circ} \mathrm{C}\right)$, muy lejos de lo predicho por los cálculos basados en una expansión adiabática irreversible $\left(\Delta T \approx-57^{\circ} \mathrm{C}\right)$. 


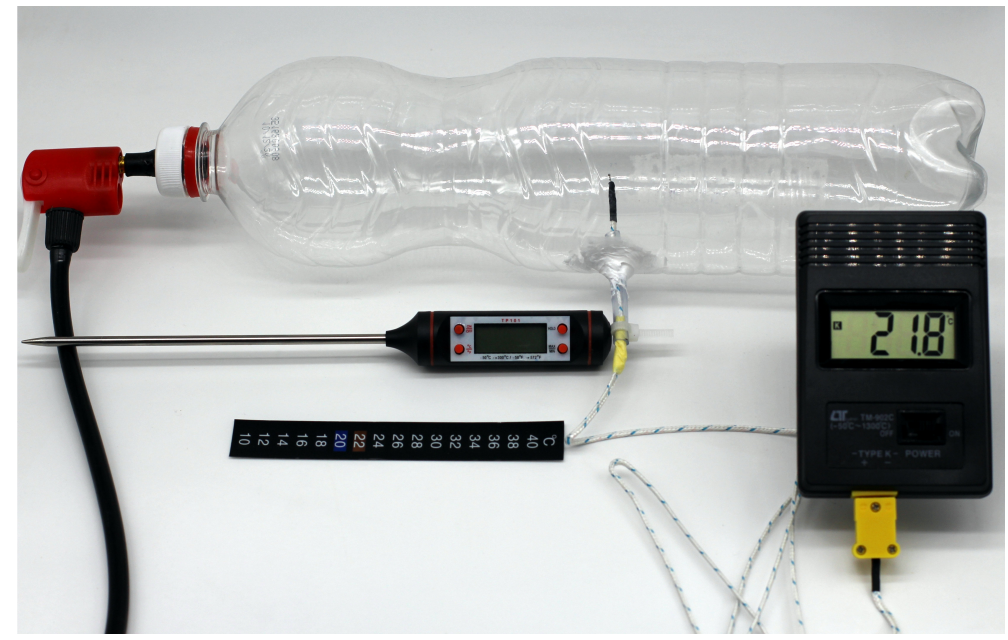

Figura 5. Montaje experimental para medir la temperatura (con un termopar estándar) en el interior de la botella. Como se describe en el texto, se obtienen resultados similares con termómetros de bajo coste (de cocina o de cristal líquido termocrómico).

Esta discrepancia se puede justificar en buena parte porque el calor liberado al condensar el vapor de etanol ( $\mathrm{y}$ el de agua, presente en el aire húmedo), contribuye notablemente a la elevación de la temperatura inmediatamente después de la descompresión (Kamcharean et al. 2016). Por otra parte, la evolución de la temperatura del interior de la botella, tras la descompresión, resulta ser muy rápida y compleja, debido a la turbulencia generada y a la interacción del sistema con las paredes y el exterior del recipiente, que se encuentran a una temperatura (temperatura ambiente) mucho mayor (Oss, Gratton, Calzà y López-Arias 2012).

En consecuencia, una sonda estándar de temperatura, con un tiempo de respuesta del orden de $0,5 \mathrm{~s}$, no resulta adecuada para medir la variación de la temperatura en este experimento; como señalan Oss et al. (2012) sería necesario utilizar una sonda con un tiempo de respuesta mucho menor $(\sim 0,001 \mathrm{~s})$ que la duración de la perturbación que se quiere medir.

\section{Algunos experimentos relacionados con la formación de la nube}

\section{Descompresión muy lenta: proceso isotérmico}

Para que baje significativamente la temperatura del interior de la botella, y condese la mayor parte del vapor de etanol tras la descompresión, no debe pasar calor del entorno hacia la botella $(Q=0$, proceso adiabático). En teoría, esto podría conseguirse aislando térmicamente el sistema, pero es mucho más sencillo efectuar una descompresión muy rápida para que no haya prácticamente tiempo a que fluya calor desde el entorno.

Ahora bien, si la descompresión se lleva a cabo de modo que el aire escape muy lentamente de la botella (que no está aislada térmicamente), a medida que el aire se expanda y realice trabajo sobre la atmósfera, pasará calor desde la atmósfera hacia la botella. Si el trabajo efectuado por el sistema es igual al calor que absorbe del entorno, la energía interna del sistema (y su temperatura) permanecerá constante $(\Delta T=0$, proceso isotérmico)

$$
\Delta U=Q-W \approx 0 ; \Delta T \approx 0,
$$

y no se observará condensación del vapor.

Para llevar a cabo este experimento será necesaria la colaboración de dos personas, una para accionar la bomba y otra para retener el tapón mientras sale lentamente el aire. Otra posibilidad es acoplar una válvula de neumático al tapón de la botella, como se muestra en la figura 2, y desenroscarlo ligeramente para que la descompresión sea muy lenta. 
En realidad, esta expansión es solo aproximadamente isotérmica, ya que tanto el aire como las paredes de la botella son malos conductores del calor y la temperatura del interior disminuye ligeramente.

\section{Utilización de otros líquidos volátiles}

Este experimento se puede realizar con otros líquidos que se evaporan con facilidad a temperatura ambiente, tales como metanol, alcohol isopropílico o acetona. Los resultados que se obtienen con ellos son similares a los del etanol, pero su manejo es algo más peligroso, ya que algunos son tóxicos (como el metanol) o muy inflamables (como la acetona o el alcohol isopropílico).

También se emplea frecuentemente el agua, a pesar de que es menos volátil que las sustancias anteriores y forma nubes menos densas al condensar. De hecho, este experimento se utiliza frecuentemente para explicar la formación de nubes en la atmósfera, mediante enfriamiento adiabático (Hewitt 1996); simplemente hay que bombear aire al interior de la botella hasta alcanzar una presión manométrica igual o mayor de 2 bar $(200 \mathrm{kPa})$ y descomprimir rápidamente a continuación. No es necesario añadir agua a la botella porque siempre hay vapor de agua en el aire atmosférico (la cantidad dependerá de la humedad relativa); al expandirse el aire, se formará una neblina de gotitas de agua. Si se desea que esta nube sea más densa, previamente habrá que añadir una pequeña cantidad de agua y agitar la botella para acelerar la evaporación y aumentar al máximo la cantidad de vapor de agua.

Otra faceta interesante del empleo del agua es que se puede llevar a cabo el experimento sin bombear aire al interior de la botella; es suficiente con comprimir y descomprimir con las manos una botella de plástico, que contenga unos $100 \mathrm{~cm}^{3}$ de agua (figura 6). La nube que se obtiene en estas condiciones es muy tenue porque las variaciones de presión y temperatura producidas $\left(\Delta p \approx 25 \mathrm{kPa}, \Delta T \approx-15^{\circ} \mathrm{C}\right)$ son menores que cuando se bombea aire $(\Delta p \approx 200$ $\left.\mathrm{kPa}, \Delta T \approx-57^{\circ} \mathrm{C}\right)$.

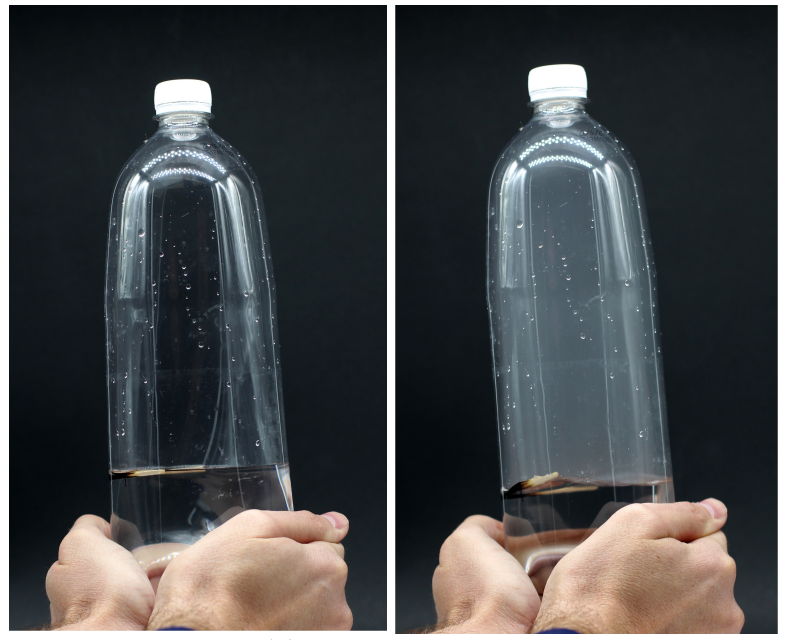

Figura 6. (Izqda.) Tras introducir una cerilla encendida, se presiona fuertemente la botella con las manos. (Dcha.) Al cesar la presión, condensa el vapor de agua y se forma una nube.
El resultado mejora si se usa agua caliente (a unos $50{ }^{\circ} \mathrm{C}$ ) y, tras agitar la botella, se arroja a su interior una cerilla encendida para que las partículas de humo, que produce al apagarse, actúen como núcleos que faciliten la condensación del vapor de agua. Este procedimiento no debe utilizarse nunca con etanol, ni con otros líquidos inflamables, porque ocurrirá una brusca deflagración al introducir la cerilla encendida en la botella.

Nótese que cuando se realiza el experimento con etanol (o cualquier otro líquido distinto al agua), el vapor de agua, presente siempre en mayor o menor cantidad en el aire atmosférico, dará lugar a una neblina que se superpondrá a la nube formada por la condensación del vapor del otro líquido.

\section{Ciclos sucesivos de compresión-expansión del vapor}

Debido a la descompresión que sufre el aire y el vapor de etanol, parte de estos gases sale proyectada fuera de la botella. Si se supone que la expansión es adiabática, e irreversible, contra la presión atmosférica $\left(p_{2}=1 \times 10^{5} \mathrm{~Pa}\right)$ y la mezcla aire-vapor de etanol es homogénea, el 
volumen $V_{2}$ ocupado por dicha mezcla $(n=0,18 \mathrm{~mol})$ inmediatamente después de la descompresión $\left(T_{2}=243 \mathrm{~K}\right)$, de acuerdo con la ecuación (6) será

$$
V_{2}=\frac{0,18 \times 8,314 \times 243}{1 \times 10^{5}}=3,6 \times 10^{-3} \mathrm{~m}^{3},
$$

es decir, aproximadamente, sale de la botella $2,1 \mathrm{~L}(3,6 \mathrm{~L}-1,5 \mathrm{~L})$, el $58 \%$ de su contenido.

Si únicamente había en la botella la cantidad máxima de vapor en el equilibrio $(0,24$ g, a 300 $\mathrm{K})$, tras la primera descompresión quedará dentro $0,42 \times 0,24=0,10 \mathrm{~g}$; tras la segunda descompresión, la masa del etanol, dentro de la botella, quedará reducida a $0,42^{2} \times 0,24=0,04$ $\mathrm{g}$, una cantidad que está cerca del límite por debajo del cual se forma una nube muy tenue, debida en parte al vapor de agua.

\section{Normas de seguridad}

Este experimento presenta una peligrosidad mínima tanto para las personas como para el medio ambiente. La única precaución a adoptar es la de mantener el etanol alejado del fuego o de chispas eléctricas, ya que sus vapores son inflamables y podrían ocasionar una explosión (Lister 2002). Tampoco es conveniente utilizar botellas de vidrio porque se podrían romper al resbalar, o caer, sobre el suelo. Y aunque el riesgo de que estalle una botella de refresco, fabricada con plástico PET, es prácticamente nulo utilizando una bomba de bicicleta, es conveniente utilizar guantes y gafas de protección.

\section{Algunos posibles planteamientos didácticos de este experimento}

Generalmente, este experimento se lleva a cabo como una demostración, ya que reúne todos los requisitos para ello. Sus resultados son muy llamativos, es sencillo y rápido, presenta escasa peligrosidad, es fácilmente reproducible, se emplean materiales baratos y fáciles de conseguir y no genera residuos. Por todo ello, el experimento se puede adaptar a diferentes niveles educativos e, incluso, se puede plantear como un pequeño trabajo de investigación en el que los estudiantes (con el consentimiento de sus familias) tendrían que realizar algunas medidas, analizar la influencia de los diversos factores que condicionan la formación de la nube y diseñar algún experimento complementario. A continuación, se señalan algunas de esas posibles tareas:

- Estimar la rapidez a la que se evapora el etanol, a una temperatura dada. Se trataría de medir la disminución de masa de un recipiente de sección constante (p. ej., un vaso cilíndrico o una cápsula Petri) lleno de etanol, durante un período de tiempo comprendido entre $24 \mathrm{~h} \mathrm{y} 48 \mathrm{~h}$, en una habitación en la que la temperatura permanezca lo más estable posible y no haya corrientes de aire. Los resultados obtenidos serán mucho más fiables si se utiliza una balanza que aprecie centésimas de gramo y se reduce el período de observación a unos 15 minutos, para que la temperatura apenas varíe (Manzur y Cardoso 2015).

- Medir las variaciones de temperatura en el interior de la botella al comprimir y descomprimir. A tal efecto, se puede realizar un montaje similar al mostrado en la figura 5 y utilizar una sonda de temperatura de bajo coste (p. ej., un termómetro de cocina o de acuario) o una tira termocrómica de cristal líquido.

- Estudiar cómo afecta la rapidez de la descompresión al descenso de temperatura que experimenta el sistema y a la consiguiente formación de la nube (expansión adiabática frente a expansión isotérmica). 
- Analizar la influencia de la presión, previa a la expansión, sobre la consistencia de la nube formada. Si no se dispone de manómetro, se puede hacer una estimación de la presión a partir del número de carreras del émbolo de la bomba.

- Observar cómo afecta la intensidad de la agitación, la cantidad de etanol y su grado de división a la densidad de la nube que se forma al descomprimir.

- Estudiar las condiciones óptimas para la obtención de una nube con vapor de agua (tanto con bombeo de aire, como sin él).

- Diseñar un experimento para determinar, de forma aproximada, la cantidad mínima de etanol necesaria para que se forme la nube $(\approx 0,04 \mathrm{~g}$, unas dos gotas de etanol, en las condiciones de presión, volumen y temperatura descritas anteriormente para este experimento).

\section{Conclusiones}

Dado que el experimento de formación de una nube en el interior de una botella atrae la atención de observadores de cualquier edad y es muy sencillo, muy rápido y muy fiable, suele utilizarse como una demostración acerca de la baja temperatura que puede alcanzarse en una expansión adiabática. Pero también puede ser un recurso didáctico muy versátil para el profesorado de Física y Química, ya que puede usarse para ejemplificar algunos conceptos básicos relacionados con el primer principio de la termodinámica, o para estudiar la dependencia de la presión de vapor de un líquido con la temperatura, o también para analizar los factores que influyen en la rapidez a la que se evapora un líquido o para discutir la importancia que tiene el tiempo de respuesta de un instrumento de medida, una característica fundamental de estos aparatos que puede pasar desapercibida en muchas ocasiones.

En esta propuesta también se describen algunos experimentos complementarios, directamente relacionados con la formación de la nube, para observar qué ocurre cuando se llevan a cabo sucesivos ciclos de compresión-expansión o para hacer una estimación de la cantidad de etanol que se encuentra en fase vapor en el momento previo a la descompresión.

Por último, se plantea la posibilidad de adaptar este experimento como un trabajo de investigación dirigido al alumnado de bachillerato, ya que no presenta riesgos si se respetan una normas elementales de seguridad, se pueden obtener buenos resultados sin emplear material de laboratorio y fomenta la imaginación y creatividad científica del alumnado.

\section{Agradecimientos}

El autor agradece al profesor Rafael García Molina de la Universidad de Murcia la ayuda prestada en el diseño y realización de las medidas de presión y temperatura que se describen en este artículo.

\section{Referencias}

Álvarez E., Manzano D. (2018) Propuesta didáctica para el empleo de la Historia de la Ciencia en la enseñanza del primer principio de la Termodinámica en Educación Secundaria. Revista de Educación de la Universidad de Granada 25, 9-28.

Barrow G. M. (1985) Quimicofísica, $4^{a}$ ed. Barcelona: Reverté.

Cortel Ortuño A. (2004) Demostraciones de termodinámica con materiales simples: Jeringas de fuego. Revista Española de Física 18 (3), 54-56.

DDB (2020) Vapor pressure of ethanol. Dortmund: Dortmund Data Bank.

Feynman R. P., Leighton R. B., Sands M. (1971) Física vol. 1: Mecánica, radiación y calor. México: Addison-Wesley Iberoamericana. 
Fishbane P. M., Gasiorowick S., Thornton S. T. (1993) Física para ciencias e ingeniería. México: Prentice-Hall Hispanoamericana.

Furió-Gómez C., Solbes J., Furió-Mas C. (2007) La historia del primer principio de la termodinámica y sus implicaciones didácticas. Revista Eureka sobre Enseñanza y Divulgación de las Ciencias 4 (3), 461-475.

Güemez J., Fiolhais C., Fiolhais M. (2007) Physics of the fire piston and the fog bottle. European Journal of Physics 28 (6), 1199-1205.

Hecht E. (1996) Física. México: Thomson.

Hewitt P. G. (1996) Conceptos de Física. México: Limusa.

Holton G. (1984) Introducción a los conceptos y teorias de las ciencias físicas. Barcelona: Reverté.

Kamcharean C., Khanchong C., Wattanakasiwich P. (2016) Revisiting the fog bottle experiment. European Journal of Physics. 37 (6), 065105

Kane J. W., Sternheim M. M. (1996) Física, 2a ed. Barcelona: Reverté.

Lister T. (2002) Experimentos de Química clásica, $2^{\mathrm{a}}$ ed. Madrid: Síntesis.

Manzur A., Cardoso J. (2015) Velocidad de evaporación del agua. Revista Mexicana de Física 61 (1), 31-34.

Oss S., Gratton L. M., Calzà G., López-Arias T. L. (2012) Fast quasi-adiabatic gas cooling: an experiment revisited. European Journal of Physics 33 (5), 1155-1165.

Pintó R. (1991) Algunos conceptos implicitos en la $1^{a}$ y $2^{a}$ leyes de la termodinámica: una aportación al estudio de las dificultades de su aprendizaje. Tesis doctoral. Universitat Autònoma de Barcelona.

Rozier S., Viennot L. (1990) Students' reasoning in thermodynamics. International Journal of Science Education 13 (2), 159-170.

Spangler S. (2017) Cloud in the bottle. https://www.youtube.com/watch?v=cXpuo3YHOn0. 